\title{
Ectopic Cornual Pregnancy-Case report and Literature Review
}

\author{
${ }^{1}$ Yakasai, IA, ${ }^{2}$ Natalia A, ${ }^{3}$ Ahmed ZD \\ ${ }^{1,2}$ Department of Obstetrics and Gynaecology, Bayero University Kano /Aminu Kano Teaching Hospital Kano, \\ Nigeria \\ ${ }^{3}$ Ectopic Cornual pregnancy-Case report and Literature review
}

\begin{abstract}
:
Background: Cornual ectopic or interstitial ectopic is a rare type of ectopic pregnancy accounting for 2-4\% of all tubal pregnancies. It is associated with serious or fatal bleeding and mortality rate up to 2-2.5\%.

Result: A 31 year old woman, $G_{3} P_{1}^{+1}$ 1Alive presented to the gynaecological emergency ward with 7 weeks amenorrhea, lower abdominal pain and vaginal bleeding on 13/06/13. A clinical diagnosis of ectopic pregnancy was made and confirmed using USS. At surgery a left cornual unruptured ectopic pregnancy was found. She had left cornual resection and salphingectomy. There were no postoperative complications.
\end{abstract}

Conclusion: It is difficult to make a clinical diagnosis of cornual ectopic pregnancy. Management is generally surgical. Our patient had Cornual resection and salphingectomy with no postoperative morbidity.

Key words: Ectopic, cornual, pregnancy

\section{Introduction}

An ectopic pregnancy is a pregnancy that implants outside the uterinecavity ${ }^{1}$.

The fallopian tube is the most common site of ectopic pregnancy, accounting for more than $95 \%$ of all ectopic pregnancies ${ }^{2}$. Cornual ectopic or interstitial ectopic is a pregnancy that implants in the intrauterine portion of the fallopian tube ${ }^{3}$. The interstitial part of the tube is about one centimeter in length and one millimeter in diameter within the uterine wall. It is a rare type of ectopic pregnancy accounting for $2-4 \%$ of all tubal pregnancies ${ }^{3}$. It is associated with serious or fatal bleeding and mortality rate up to $2-2.5 \%{ }^{3}$. Early diagnosis is important, as it not only allow preventing deadly complications, but allows varied choice of treatment options and possibility of conserving fertility.

Transvaginal ultrasonography, serum $\beta h C G$ determination and laparoscopy have proven diagnostic value in the evaluation of symptomatic women with suspected ectopic pregnancy. In normal pregnancy, $\beta h C G$ concentrations rise predictably exponentially with doubling time of 48 hours, in contrast, in abnormal pregnancies, its level rises at slower rate than normal or with linear pattern. The earliest normal intrauterine sac is seen at 4 weeks of gestation using transvaginal ultrasound with a corresponding $\beta h C G$ level of 1000-2000 $\mathrm{iu} / \mathrm{L}$, while at 5 weeks using transabdominal ultrasound with a corresponding $\beta h C G$ level of $6000-6500 \mathrm{liu} / \mathrm{l}^{1,2}$. Surgery is the main stay of treatment ${ }^{4}$.We report a case of unruptured cornual ectopic pregnancy.

\section{Case Report}

A 31 year old woman, $\mathrm{G}_{3} \mathrm{P}_{1}{ }^{+1}$ 1Alive presented to the gynaecological emergency ward of Aminu Kano Teaching Hospital on 13/06/13. Her last menstrual period was on 19/4/ 13 and the estimated gestational age was seven weeks one day. She complained of non-radiating, dull lower abdominal pain which was accompanied with bleeding per vaginam. There was no dizziness or syncopal attacks. Her first pregnancy in 2011 ended with caesarean section at term due to cephalo-pelvic disproportion and she was delivered of a live male baby. The second pregnancy ended as spontaneous miscarriage at six weeks of gestation in 2012. She had manual vacuum aspiration. The postabortal period was uncomplicated.

On general examination, she was in pains but not pale and anicteric. The pulse rate was 78 beats per minute and her blood pressure was $110 / 70 \mathrm{mmHg}$. The abdomen was not distended and mild tenderness was elicited in the right iliac fossa with no guarding. Pelvic examination revealed normal external genitalia, closed cervix and positive cervical excitation tenderness. The uterus was bulky and tender and examining finger was stained with blood.

She had a positive urine pregnancy test. Transabdominal 2-dimensional ultrasound revealed an empty uterus and thickened endometrial plate of 10 millimeters. There was a roundish well encapsulated mass measuring $41 \times 42.9 \mathrm{~mm}$, eccentrically positioned at the upper portion of the uterus 14 millimeters away from the lateral edge of the uterine cavity with no fetal pole seen within it. There was no fluid collection in a pouch of Douglas.

An assessment of ectopic pregnancy was made and the patient was counseled for exploratory laparotomy. The intra operative findings were: clean abdomino-pelvic cavity, and bulky uterus. The right cornua 
was distended by a mass 5 by 6 centimeters(Fig 1). Right and left ovaries and left tube were normal. The right tube was dilated and knotted. The estimated blood loss was 200 milliliters. She had right Cornual resection and salphingectomy. Histopathological evaluation of the specimen confirmed the diagnosis of ectopic pregnancy. The postoperative period was uncomplicated and she was discharged home on the fifth day. She was advised to avoid progestational contraceptives and advised on caesarean section in subsequent pregnancies to reduce the risk of uterine rupture.

\section{Discussion}

Unruptured ectopic pregnancy is a rare event associated with high risk of rupture followed by life threatening haemorrhage and high mortality. Ruptured ectopic pregnancy presents with classical symptoms of delayed menses, vaginal bleeding and haemorrhagic shock due to severe intraperitoneal bleeding ${ }^{2}$. It is important to diagnose cornual pregnancy before it ruptures. Transvaginal ultrosonography is the most useful diagnostic method for establishing the diagnosis. The sac usually eccentrically positioned and may be surrounded by thin myometrium and away from the endometrium. Doppler ultrasound can help in diagnosis by showing increase vasculature around the gestational sac ("ring of fire") $)^{2}$. However, similar picture can also be seen in early pregnancy, bicornuate uterus or in a fibroid uterus ${ }^{3}$. Therefore diagnosis is often challenging and need experienced radiologist. Laparoscopy is also challenging, as small ectopic masses can be missed or large ectopic masses can be misdiagnosed with uterine fibroids or bicornuate uterus ${ }^{3}$. In this case the diagnosis was based on clinical findings and was augmented by urine pregnancy test and transabdominal ultrasound. In this patient using 2-dimensional transabdominal ultrasound for diagnosis was difficult as the sac was eccentrically located and differentiation between eccentric intrauterine and Cornual pregnancy was usually based on findings of an empty uterine cavity, and gestational sac located 14 millimeters away from the most lateral edge of the uterine cavity, just above the uterine fundus.

The common procedures performed in a management of cornual pregnancy is cornual resection, cornuotomy, cornual resection with salpingectomy and hysterectomy $y^{5,6}$. The procedure is difficult and may be complicated by a severe bleeding due to good blood supply to the uterine cornuae. Use of diathermy or harmonic scalpel can help to reduce bleeding ${ }^{6}$. The procedure can be performed either through laparotomy or laparoscopically, provided a skilled surgeon and equipment are available. Laparoscopical approach is associated with less intra operative bleeding, less postoperative pain, faster recovery, shorter hospital stay, few postoperative adhesions, preservation of fertility and less risk of recurrent ectopics ${ }^{7}$. However, in our center operative laparoscopic equipment is not yet available, hence was not offered to our patient. If the size of the sac is less then $4 \mathrm{~cm}, \beta \mathrm{hCG}$ is less then $3000 \mathrm{IU}$, fetal heartbeat is absent, patient is haemodynamically stable and no contraindications to Methotrexate, then medical treatment is the another option. Methotrexate can be given systematically or injected directly into the ectopic sac under trasvaginal ultrasound guidance ${ }^{3}$.

Our patient was not offered a medical treatment, as the size of the sac was more than $4 \mathrm{~cm}$. Therefore, right Cornual resection and salphingectomy was considered to prevent rupture with torrential haemorrhage and the recurrence of ectopic pregnancy in the ipsilateral tube (which was grossly dilated and the other one was normal).

Following surgery the subsequent intrauterine pregnancy rate is $49.3 \%{ }^{1}$.

Generally the management is tailored according to the general condition of the patient, hemodynamic status, gestational age, presence of rupture, fertility desire $\beta \mathrm{hCG}$ level and most importantly availability of instruments and physician's experience and preference.

\section{Conclusion}

It is difficult to make a clinical diagnosis of Cornual ectopic pregnancy. Management is generally surgical. Our patient had Cornual resection and salphingectomy with no postoperative morbidity.

\section{References}

[1]. Lukas DK. Ectopic Pregnancy. In: Shaw RW editor. Gynaecology. $3^{\text {rd }}$ ed. Londom (UK): Elsevier Science Limited; 2003. p. $371-86$

[2]. Ectopic Pregnancy. In: Speroff L, Marc AF editors. Clinical gynaecologic Endocrinology and Infertility. $7^{\text {th }}$ ed. New York (NY): Lippincott Williams \& Wilkins. 2005; 1: p.1276-96.

[3]. M Shendy, R Atalla. Modern management of cornual ectopic pregnancy. Available from: http://www.intechopen.com/books.

[4]. D Larrain, F Marengo, N Bourdel, P Jaaffeux, B Cuvelier, J Pouly et al. Proximal ectopic pregnancy: a descriptive general population- based study and results of different management options in 86 cases. Fertil Steril. 2011; 95(3): 867-71.

[5]. J Hwang, J Lee, N Lee, L lee. Open cornual resection versus laparoscopic cornual resection in patients with interstitial ectopic pregnancies. European J Obstet Gynaecol Reproduct Biol. 2011; 156:78-82.

[6]. E Lee, H Hahn, B Park, D Ro, J Kim, Y Kim. Single-port laparoscopic cornual resection for a spontaneous cornual ectopic pregnancy following ipsilateral salpingectomy. Fertil Steril. 2011; 96(2): 106-9.

[7]. S Uccella, A Cromi, G Bogani, F Ghezzi. Laparoscopic management of cornual pregnancy: minimally invasive surgery reduced the risk for bleeding. Am J Obstet Gynaecol, 2011; 205(1); 579. 